\title{
Performance and operation of the ATLAS Resistive Plate Chamber system in LHC Run-1
}

\author{
D. Boscherini ${ }^{a *}$ on behalf of the ATLAS Muon Collaboration \\ ${ }^{a} I N F N$, \\ Via Irnerio 46, 40126 Bologna, Italy \\ E-mail: davide.boscheriniebo.infn.it
}

\begin{abstract}
The barrel region of the ATLAS muon spectrometer is instrumented with a Resistive Plate Chamber (RPC) system covering the pseudo-rapidity range $|\eta|<1.05$ with a detector surface of almost $4000 \mathrm{~m}^{2}$. The RPCs, providing the first level trigger signal and the track coordinate in the non-bending plane for the candidate muons, have played a fundamental role in the physics studies carried out by ATLAS, culminated with the discovery of the Higgs boson. During the LHC Run-1 the RPC have shown excellent performance up to the maximum instantaneous luminosity of $0.7 \times$ $10^{34} \mathrm{~cm}^{-2} \mathrm{~s}^{-1}$, corresponding approximately to $70 \%$ of the design value. The detector operation in the challenging background and pileup conditions of the LHC environment are presented together with the problems encountered and their corresponding solutions. The plans for the maintenance and consolidation of the ATLAS RPC system during the current LHC shutdown, in view of the increased luminosity expected in Run-2, are also presented.
\end{abstract}

KEYWORDS: RPC; trigger; performance; ATLAS; LHC.

${ }^{*}$ Corresponding author. 


\section{Contents}

1. The RPC System of the ATLAS Detector

2. Detector and Trigger Performance during LHC Run-1 근

3. Operational Issues

4. Preparation for LHC Run-2

4.1 Consolidation Works

4.2 Minor Upgrades

5. Conclusions 8

\section{The RPC System of the ATLAS Detector}

The ATLAS detector [ [I] is a multipurpose particle physics apparatus, $44 \mathrm{~m}$ long and $25 \mathrm{~m}$ high. Moving from the interaction vertex outwards, it is equipped with an inner tracker immersed in a $2 \mathrm{~T}$ solenoidal field, a liquid argon electromagnetic calorimeter, a scintillator-steel hadronic calorimeter and a muon spectrometer surrounding the whole detector and exploiting the magnetic field of an air-core toroid system.

The muon spectrometer is built with four different detector technologies [2]]: Monitored Drift Tubes and Cathode Strip Chambers measure with high precision the bending coordinate up to $|\eta|<2.7$, Resistive Plate Chambers in the barrel region $(|\eta|<1.05)$ and Thin Gap Chambers in the endcap regions $(1.05<|\eta|<2.4)$ also provide the non-bending coordinate $\phi$ and the trigger signal up to $|\eta|<2.4$.

The RPC chambers, made of two independent detector layers, are arranged in 3 concentric cylindrical shells at approximately 7-10 m radius and are operated in a toroidal magnetic field of about $0.5 \mathrm{~T}$. The cross-view of the an RPC is shown in Fig. [1: it is made of bakelite gas volumes with a $2 \mathrm{~mm}$ gap, flushed with a gas mixture of $\mathrm{C}_{2} \mathrm{H}_{2} \mathrm{~F}_{4}(94.7 \%)$ : iso- $\mathrm{C}_{4} \mathrm{H}_{10}(5.0 \%): \mathrm{SF}_{6}(0.3 \%)$ operated in avalanche mode at $4.8 \mathrm{kV} / \mathrm{mm}$ with automatic correction for temperature and pressure variations with respect to the reference values of $24{ }^{\circ} \mathrm{C}$ and 970 mbar, respectively. Readout panels with strips 2.3-3.5 $\mathrm{cm}$ wide are used to measure two orthogonal coordinates (pseudo-rapidity, $\eta$, and azimuth, $\phi$ ). The full system is made of about 3600 gas volumes, covering a surface of 3650 $\mathrm{m}^{2}$ with about 370,000 readout channels.

The collision events are selected by ATLAS using a three-level trigger system to reduce the interaction rate from $\sim 1 \mathrm{GHz}$ to $\sim 400 \mathrm{~Hz}$ affordable by storage and reconstruction. The level-1 is a hardware system based on dedicated electronics using only data from a selected region of the detector, while level-2 and event-filter, commonly called High Level Trigger (HLT) are software 


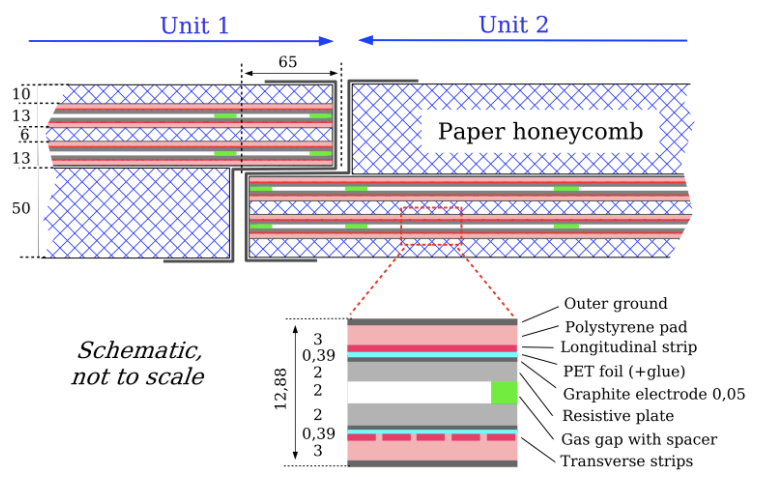

Figure 1. Cross-section through a RPC, where two units are joined to form a chamber. The detector is made of plastic laminate electrodes spaced by a $2 \mathrm{~mm}$ gas gap. Independent measurements of the two perpendicular coordinates are provided by each of the two layers [䧃].

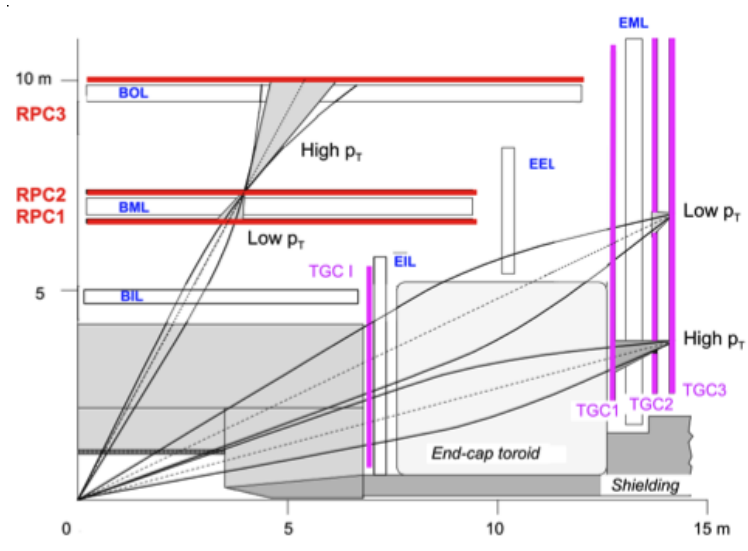

Figure 2. Schematic of the muon level-1 trigger system. The three RPC planes are shown in red. The middle plane, RPC2, acts as the reference (pivot) plane for the barrel region and its coincidence with a selected region of the inner layer (RPC1) provides the low-pt trigger. The additional coincidence with the outer layer (RPC3) will eventually produce the high-pt trigger [1]].

systems running on PCs with access to the full detector data. The RPCs are the unique detector providing a level-1 trigger signal for muons in the barrel region [3]. The schematic layout of the muon level-1 trigger is shown in Fig. 2 . In the barrel the trigger logic is implemented starting from a reference plane, the middle RPC layer, and requiring a coincidence with the innermost RPC layer to obtain the low-pt trigger. By adding the coincidence with the outermost RPC layer the high-pt trigger is eventually obtained.

\section{Detector and Trigger Performance during LHC Run-1}

During LHC Run-1 ATLAS has collected an integrated luminosity of $25 \mathrm{fb}^{-1}$. A maximum instantaneous luminosity of $0.7 \times 10^{34} \mathrm{~cm}^{-2} \mathrm{~s}^{-1}$ was reached with an average number of collisions per bunch-crossing (pile-up) up to 40 , well above the maximum value of 25 expected by design. The RPCs have shown a very good behavior over the whole Run-1, with a fraction of active readout channels generally above $97 \%$. The dead channels were mainly due to disabled trigger towers and 


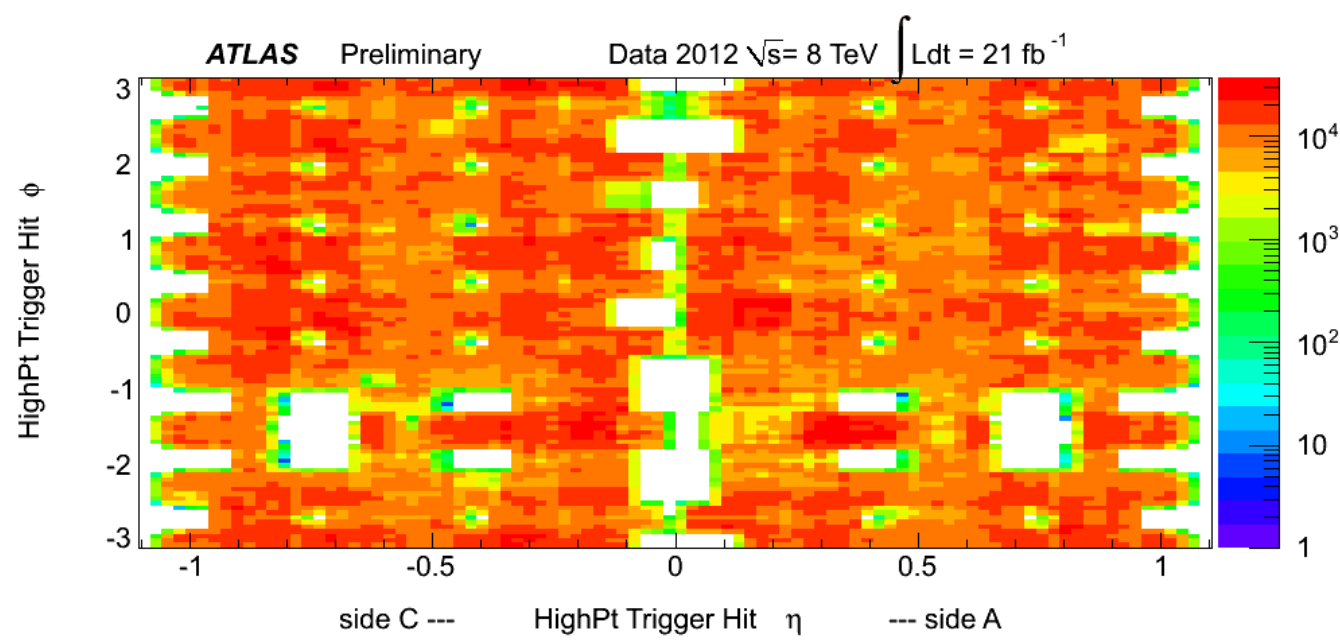

Figure 3. Spatial coincidence between $\eta$ and $\phi$ RPC pivot strips generating a high-pt trigger in terms of the strip center coordinates integrated over 2012 data taking period. Side C and side A denote the negative- and positive-z side of the detector, respectively [四].

gas volumes disconnected because of leaks or high currents. The good data quality fraction, i.e. the fraction of data usable for the physics analysis, has also reached the excellent value of $99.8 \%$ averaged on the whole 2012 data taking.

Fig. 3 shows the spatial coincidence between $\eta$ and $\phi$ strips in the middle RPC plane generating a high-pt trigger signal integrated over 2012 data taking period. The uniform population over all the spectrometer confirms the stable behavior of the detector. The empty regions are due to not instrumented areas needed for services, toroid support feet and toroid coils.

The efficiency of the gas volumes and of the detector (i.e. including front-end electronics effects) has been measured and is shown in Fig. 4 for the whole 2012 running. The detector efficiency includes also the effects of the dead channels accepted in the qualification process at the time of chamber construction. The distribution of the readout hit time is shown in Fig. 5 for all the 2012 data taking. A standard deviation slightly above $3.5 \mathrm{~ns}$ has been found, a value expected before applying the offline corrections and consistent with the 2011 measurement.

The level-1 muon barrel trigger efficiency as a function of $p_{T}$ on a luminosity fill towards the end of the 2012 data taking is shown in Fig. 6 as measured from the offline data quality monitoring. The efficiency is measured using offline reconstructed combined muons and an independent trigger based on jets and missing transverse energy. The lower trigger efficiency for the three high-pt thresholds is due to the reduced RPC detector coverage in the outer planes, due to the ATLAS feet support structure. The efficiency results for all the thresholds are very close to the geometrical acceptance values, confirming the very good detector performance.

In order to check possible effects on the trigger from the pile-up conditions, a measurement of the trigger effciency versus the number of interactions per bunch-crossing has been done with the tag-and-probe method on $\mathrm{Z} \rightarrow \mu^{+} \mu^{-}$candidate events. In Fig. 7 the efficiency of the Event Filter trigger with $p_{T}>24 \mathrm{GeV}$ is shown and a very stable behavior is visible up to the highest pileup values reached so far. The efficiency includes the geometric acceptance of the Level-1 trigger chambers. 


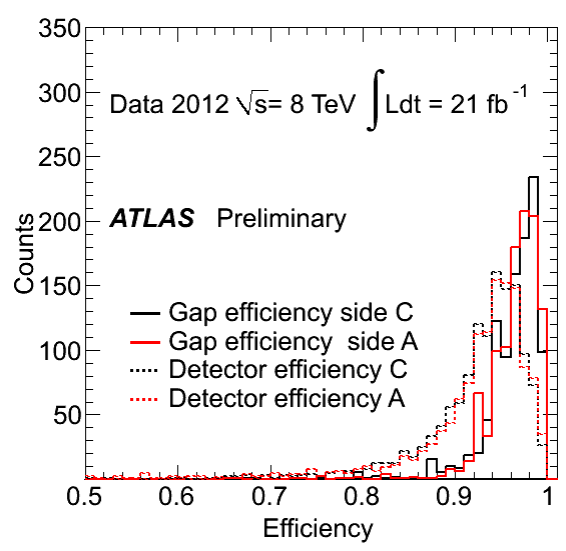

Figure 4. Distribution of the measured 2012 RPC gas volume efficiencies defined by the positive response of at least one of the two views and detector efficiency defined by the positive response of the single views. The efficiency is measured using standalone RPC track projection obtained removing the layer under test and using cluster hits matched with a muon combined track $(\Delta \eta<0.1$ and $\Delta \phi<0.1)$ [ [⿴囗十 ].

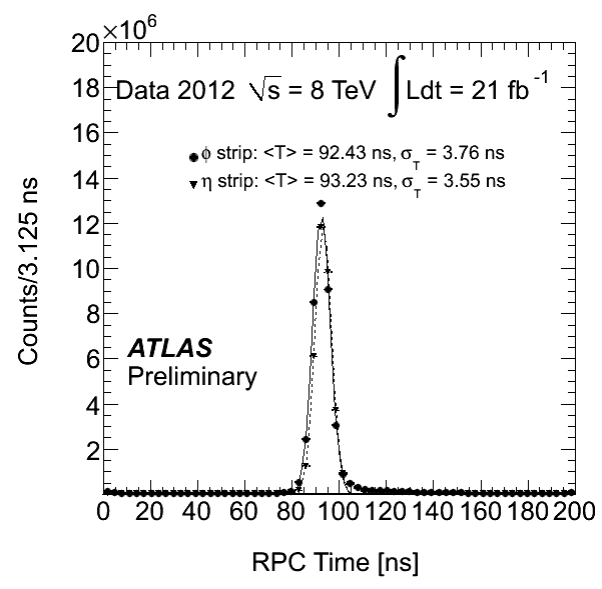

Figure 5. Distribution of 2012 RPC time in readout hits for both views. The RPC cluster hit time is defined as the minimum time of adjacent hits. Only cluster hits matched with a muon combined track $(\Delta \eta<0.1$ and $\Delta \phi<0.1)$ are considered. The signal propagation along the strip is subtracted but off-line time corrections are not applied [四].

The ATLAS RPCs aging qualification test was done assuming $100 \mathrm{~Hz} / \mathrm{cm}^{2}$ for 10 years of LHC running. This included a safety factor 5 with respect to $20 \mathrm{~Hz} / \mathrm{cm}^{2}$ of the maximum expected rate from the simulation result. Fig. 8 shows, for each region of the middle layer of the barrel spectrometer, the background rate measured in 2012 at a luminosity of $0.6 \times 10^{34} \mathrm{~cm}^{-2} \mathrm{~s}^{-1}$, extrapolated to $1.0 \times 10^{34} \mathrm{~cm}^{-2} \mathrm{~s}^{-1}$ at a center of mass energy of $14 \mathrm{TeV}$ (the LHC design values). At large $\eta$ the rates are above the expectations and will certainly exceed the $100 \mathrm{~Hz} / \mathrm{cm}^{2}$ during HL-LHC. A few proposals to overcome or mitigate this issue are discussed in [8]. 


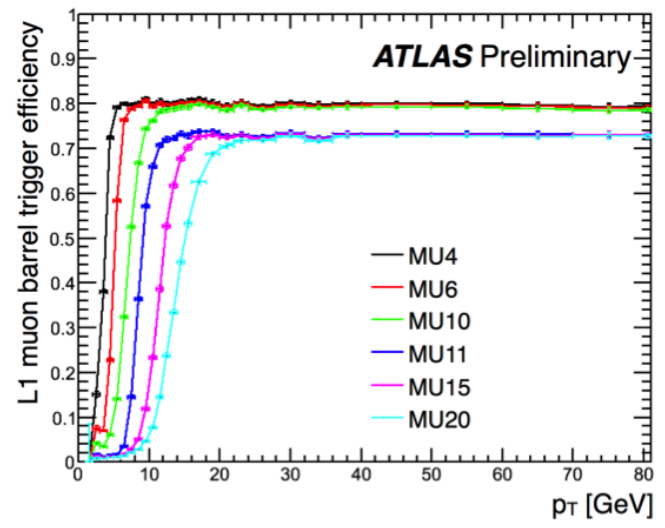

Figure 6. Level-1 muon barrel trigger efficiency as a function of $p_{T}$, for the six trigger thresholds. MU4, MU6, MU10 are the low-pt thresholds (muons selected with the two inner RPC stations), while MU11, MU15, MU20 are the high-pt thresholds (low-pt triggered muons confirmed with the third outer RPC station). These results are obtained from the offline data quality monitoring for the LHC fill 3203, 20-21 October 2012 [5].

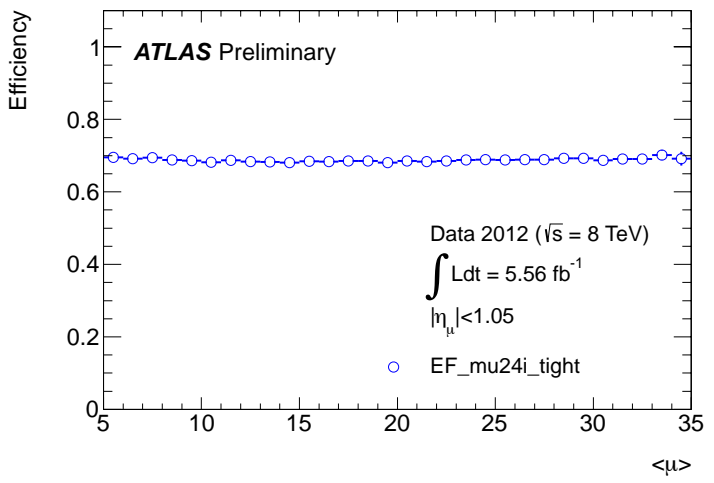

Figure 7. Efficiency in the barrel region of the Event Filter trigger with respect to offline reconstructed isolated muons as a function of the average number of interactions per bunch crossing $(<\mu>)$. The trigger requires an isolated muon with $p_{T}>24 \mathrm{GeV}$ at the Event Filter. The vertical error bars represent the statistical errors [6].

\section{Operational Issues}

Despite the very successful data taking experienced in Run-1, a few operational issues had to be faced and here below are briefly reported:

HV sparking connectors. Until 2011 the breakdown of the insulator was observed in a few HV connectors (60 out of 8000). This was traced back to an inappropriate cooling process at the manufacturer for a batch of insulators installed in the connector on the rack-side of the cables. No failure was observed after the refurbishment of the rack-side connectors.

Power system connection failures. The melting of connectors in the $48 \mathrm{~V}$ power distribution occurred in four occasions [1]] due to the degradation of the electrical contact. The power distribution scheme has been rebuilt changing from a daisy-chain to a star connection and exploiting the 


\begin{tabular}{|c|c|c|c|c|c|c|c|c|c|c|c|c|c|c|c|c|c|c|c|c|c|c|c|}
\hline \multirow{2}{*}{$\begin{array}{l}\text { Sector } \\
\Phi \text { Id. }\end{array}$} & \multirow[b]{2}{*}{-7} & \multirow[b]{2}{*}{-6.2} & \multirow[b]{2}{*}{-6.1} & \multirow[b]{2}{*}{-5} & \multirow{2}{*}{\multicolumn{10}{|c|}{ 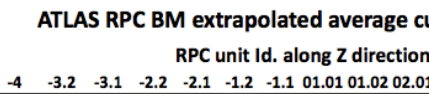 }} & rren & der & sity & \multicolumn{5}{|c|}{ ATLAS preliminary } & \multirow[t]{2}{*}{$\begin{array}{l}\text { Average } \\
\left(\mu \mathrm{A} / \mathrm{m}^{2}\right)\end{array}$} \\
\hline & & & & & & & & & & & & & & & 02.02 & 03.01 & 3.02 & 4 & 5 & 06.01 & 06.02 & 7 & \\
\hline 01.01 & 12 & 13 & 11 & 11 & 9 & 6 & 4 & 5 & 4 & & 3 & 3 & 3 & 4 & 5 & 5 & 5 & 8 & 11 & 10 & 11 & 9 & 7 \\
\hline 01.02 & & 11 & 11 & 11 & 8 & 6 & 5 & 5 & 5 & & 3 & 3 & 3 & 5 & 5 & 5 & 6 & 8 & 12 & 11 & 12 & 11 & 7 \\
\hline 2 & & 5 & 8 & 7 & 5 & 4 & 4 & 3 & 3 & 2 & 2 & 2 & 2 & 3 & 3 & 4 & 4 & 5 & 8 & 7 & 6 & & 4 \\
\hline 03.01 & & 11 & 11 & 11 & 8 & 6 & 5 & 5 & 4 & 3 & 3 & 3 & 3 & 5 & 5 & 5 & 5 & 8 & 11 & 10 & 11 & & 7 \\
\hline 03.02 & & 11 & 11 & 12 & 8 & 6 & 5 & 6 & 5 & 3 & 3 & 3 & 3 & 5 & 5 & 6 & 6 & 8 & 11 & 10 & 11 & & 7 \\
\hline 4 & & 4 & 7 & 7 & 6 & 4 & 4 & 3 & 3 & 2 & 2 & 2 & 2 & 3 & 3 & 4 & 4 & 6 & 7 & 7 & 6 & & 4 \\
\hline 05.01 & 11 & 8 & 8 & 10 & 6 & 4 & 4 & 5 & 4 & 3 & 3 & 3 & 3 & 4 & 6 & 4 & 5 & 7 & 9 & 7 & 7 & 8 & 6 \\
\hline 05.02 & 11 & 9 & 9 & 8 & 5 & 4 & 5 & 5 & 5 & 3 & 3 & 3 & 3 & 4 & 5 & 4 & 5 & 6 & 9 & 10 & 9 & & 6 \\
\hline 6 & & 5 & 7 & 8 & 6 & 4 & 4 & 3 & 3 & 3 & 2 & 2 & 2 & 3 & 3 & 4 & 4 & 6 & 7 & 8 & 7 & & 5 \\
\hline 07.01 & & 11 & 10 & 11 & 8 & 6 & 5 & 5 & 4 & & 3 & 3 & & 4 & 5 & 5 & 6 & 7 & 10 & 10 & 11 & 10 & 7 \\
\hline 07.02 & 10 & 12 & 10 & 9 & 5 & 5 & 4 & 5 & 4 & & 3 & 3 & & 4 & 5 & 5 & 6 & 4 & 12 & 11 & 10 & 11 & 6 \\
\hline 8 & & 4 & 8 & 7 & 6 & 4 & 3 & 3 & 3 & 2 & 2 & 2 & 2 & 3 & 3 & 3 & 4 & 6 & 7 & 7 & 6 & & 4 \\
\hline 09.01 & & 12 & 10 & 11 & 8 & 6 & 5 & 5 & 4 & & 2 & 3 & & 4 & 5 & 5 & 5 & 8 & 10 & 10 & 11 & 8 & 7 \\
\hline 09.02 & 8 & 12 & 10 & 11 & 7 & 6 & 4 & 4 & 4 & & 2 & 3 & & 4 & 4 & 4 & 5 & 7 & 11 & 10 & 11 & 8 & 7 \\
\hline 10 & & 5 & 8 & 8 & 5 & 3 & 3 & 3 & 3 & 2 & 2 & 2 & 2 & 3 & 3 & 3 & 3 & 5 & 7 & 7 & 7 & & 4 \\
\hline 11.01 & 9 & 11 & 9 & 9 & 6 & 4 & 3 & 4 & 3 & & 2 & 2 & & 3 & 3 & 4 & 4 & 6 & 9 & 8 & 10 & 7 & 5 \\
\hline 11.02 & 9 & 7 & 7 & 6 & 4 & 3 & 3 & 2 & 2 & & 1 & 1 & & 2 & 2 & 3 & 3 & 4 & 6 & 6 & 7 & 9 & 4 \\
\hline 12 & & & & & & 4 & 4 & 3 & 3 & 2 & 1 & 1 & 2 & 3 & 3 & 4 & 4 & & & & & & 3 \\
\hline 13.01 & 12 & 11 & 10 & 9 & & 5 & 4 & 4 & 3 & 2 & 2 & 2 & 2 & 3 & 4 & 3 & 5 & & 9 & 10 & 11 & 7 & 6 \\
\hline 13.02 & 12 & 11 & 10 & 8 & & 4 & 4 & 4 & 3 & 2 & 2 & 2 & 2 & 3 & 4 & 4 & 5 & & 8 & 9 & 11 & 7 & 5 \\
\hline 14 & & & & & & 5 & 5 & 3 & 2 & 2 & 1 & 1 & 2 & 3 & 3 & 5 & 5 & & & & & & 3 \\
\hline 15.01 & 7 & 7 & 7 & 6 & 4 & 3 & 3 & 3 & 2 & & 2 & 1 & & 2 & 2 & 3 & 4 & 4 & 6 & 6 & 7 & 7 & 4 \\
\hline 15.02 & 10 & 7 & 6 & 9 & 6 & 5 & 4 & 4 & 3 & & 2 & 2 & & 3 & 4 & 4 & 5 & 6 & 9 & 7 & 8 & 7 & 5 \\
\hline 16 & & 5 & 8 & 8 & 6 & 4 & 3 & 2 & 3 & 2 & 2 & 2 & 2 & 3 & 2 & 3 & 4 & 6 & 8 & 8 & 6 & & 5 \\
\hline $\begin{array}{l}\text { Average } \\
\left(\mu \mathrm{A} / \mathrm{m}^{2}\right)\end{array}$ & 10 & 8 & 9 & 9 & 6 & 5 & 4 & 4 & 3 & 2 & 2 & 2 & 2 & 3 & 4 & 4 & 5 & 6 & 9 & 8 & 8 & 9 & 5 \\
\hline
\end{tabular}

Figure 8. RPC middle layer background map measured at a luminosity of $0.6 \times 10^{34} \mathrm{~cm}^{-2} \mathrm{~s}^{-1}$ and extrapolated to $1.0 \times 10^{34} \mathrm{~cm}^{-2} \mathrm{~s}^{-1}$ at a center of mass energy of $14 \mathrm{TeV}$. The horizontal axis represents the RPC units in the $\mathrm{z}$ direction, the vertical axis represents the spectrometer sectors or half-sectors in the azimuthal direction [四].

redundancy of the connectors on the crates. The monitoring of the current driven by the $48 \mathrm{~V}$ lines has been implemented in the Detector Control System. No failure has been observed anymore.

Disconnected gaps. At the end of 2012, 2.5\% of the gas volumes were disconnected from the HV lines due to gas problems, high gap currents or electrical problems [ 9 ]. A plan for recovering most (and possibly all) of the the gaps is being followed during the current long shutdown.

Cavern temperature distribution. The top side of the barrel spectrometer has been operated with a cavern temperature above $24{ }^{\circ} \mathrm{C}$, maximum value accepted for a long-term safe operation of the detector. The gas volumes with a temperature exceeding $26{ }^{\circ} \mathrm{C}$ were operated at a reduced $\mathrm{HV}$ ( $100 \mathrm{~V}$ below the nominal value, with automatic correction for temperature and pressure variations) to avoid an early uprising of aging effects. Two attempts made by ATLAS to re-distribute the cooling air flow only mitigated the problem; a more substantial upgrade of the cavern cooling system is planned in LS1. Anyway, no evidence of temperature effects has been observed on the chamber operation.

Gas leaks. About $4 \%$ of gas volume nozzles developed fractures or complete breakups due to the mechanical stress induced by the piping (see Fig. (9). Despite the leaks, most volumes could be normally operated at the cost of a higher flow of fresh gas needed by the system. A massive repair campaign is ongoing during the current shutdown, aiming to fix all the detected leaks. The details on the repair techniques can be found in [10].

Weak grouding connections. Weak grounding connection between readout panels and frontend electronics affected about $6 \%$ of the panels which were operated with a harder discrimina- 


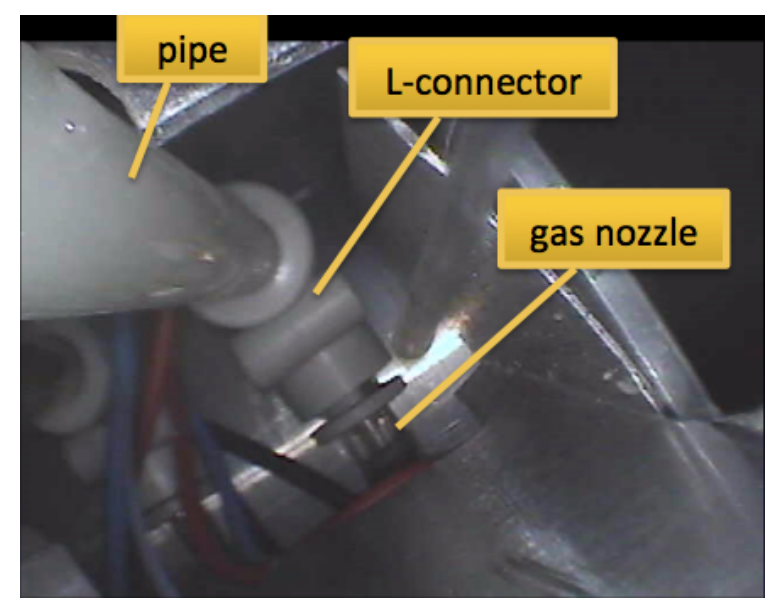

Figure 9. Detail of the gas distribution to the RPCs as seen through the endoscope used for the gas repairs. The pipe is connected to the gas volume inlet via a L-shaped connector.

tion threshold. The enhancement of the Faraday cage in the affected chambers (installing custom grounding plates) fixed or mitigated the problem.

\section{Preparation for LHC Run-2}

Several maintenance and consolidation works are being carried out during the current LHC shutdown in preparation for Run-2.

\subsection{Consolidation Works}

Gas flow increase and re-distribution. The gas re-circulation flow in 2012 was run at an exchange rate of one volume every 2.5 hours to be compared with the value of one volume per hour foreseen at the design luminosity of $1 \times 10^{34} \mathrm{~cm}^{-} 2 \mathrm{~s}^{-1}$. An additional pump will be installed in the system to reach the nominal flow. Further increase of the gas flow might be needed at higher luminosities. The gas input impedances installed on each chamber will also be replaced to better match the measured background distribution.

Gas monitoring improvement. For an early spot of the leaks and a better monitoring of the gap conditions the granularity of the flow monitoring will increase from one measurement per gas line (128) to one measurement per chamber-layer (1200).

Gas leak repairs. A check-up of the full system has been recently completed. Many unidentified leaks have been found explaining the amount of fresh gas needed by the system (and not matching the rough measurement from the flowmeters). Most of the detected leaks are due to cracked or completely broken gas inlets and an intensive repair campaign is ongoing all along LS1 as reported in [10].

\subsection{Minor Upgrades}

Instrumentation of the detector elevator shafts. The barrel trigger acceptance will be increased by $0.9 \%$ with the installation of four additional muon stations (see Fig. 10 left), two per ATLAS 

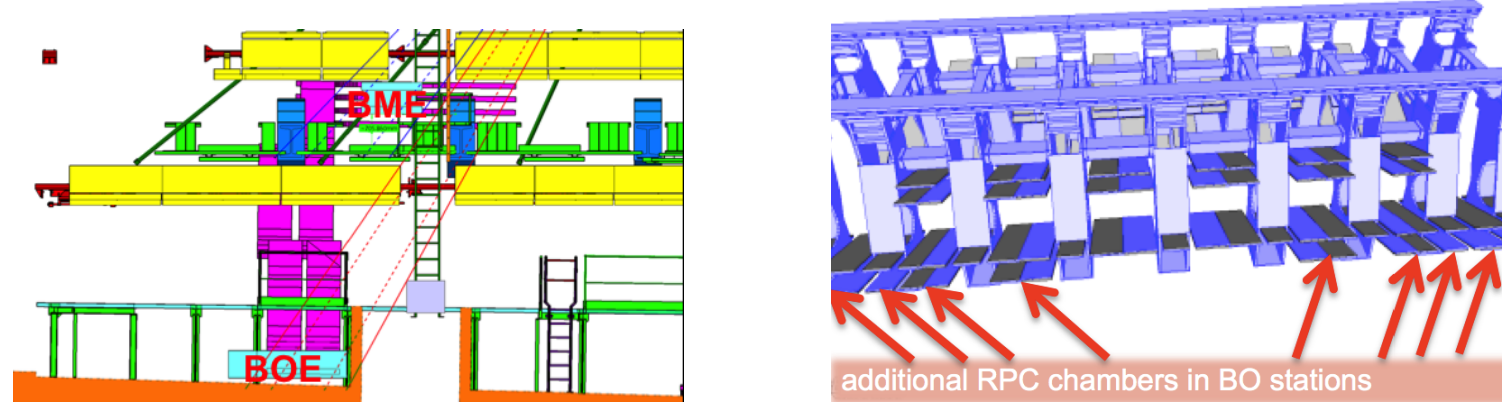

Figure 10. Left: sketch of the bottom part of the ATLAS barrel spectrometer with the newly installed chambers. Right: perspective view of the additional chambers under commissioning in the region of the toroid support feet.

side, which will cover non instrumented regions needed to access the innermost zones of the ATLAS apparatus. New generation 1mm-gap RPCs will be used in the middle chambers, as an early study for the development of RPCs to be used in more severe background conditions.

Commissioning of additional chambers in the toroid support feet. Twenty RPC chambers (see Fig.10 right), installed since 2007 to fill the acceptance holes due to the toroid support feet, were not equipped with the trigger electronics until recently, because of the lack of funding. The chambers are therefore being commissioned providing an additional increase of $2.8 \%$ in the barrel trigger acceptance.

\section{Conclusions}

The ATLAS RPCs have operated very successfully during LHC Run-1, maintaining high efficiency and high data quality over the whole period. A few technical issues have been promptly tackled and absorbed by the system redundancy without sizeable effects on the data taking. The RPCs, being the only system with trigger capability in the barrel region of the muon spectrometer, have played a key role in the discovery of the Higgs boson [12]. The consolidation and upgrade works being carried out on the system will ensure the ATLAS RPCs will be able to exploit at best the luminosity delivered by LHC in the next years.

\section{References}

[1] ATLAS Collaboration, The ATLAS Experiment at the CERN Large Hadron Collider, 2008 JINST 3 $\$ 08003$

[2] ATLAS Muon Collaboration, ATLAS Muon Spectrometer Technical Design Report, CERN/LHCC/97-22, (1997).

[3] F. Anulli et al., The Level-1 Trigger Muon Barrel System of the ATLAS experiment at CERN, 2009 IINST 4 P04010.

[4] G.Chiodini et al., RPC plots for approval, ATL-COM-MUON-2014-009 (2014). 
[5] M. Corradi et al., L1 Barrel Muon Trigger Efficiency with 2012 Data, ATL-COM-DAQ-2014-007 (2014).

[6] ATLAS Muon Trigger Public Results web page, https://twiki.cern.ch/twiki/bin/view/AtlasPublic/MuonTriggerPublicResults

[7] ATLAS Muon Public Results web page, https://twiki.cern.ch/twiki/bin/view/AtlasPublic/MuonPublicResults

[8] G. Aielli, High Luminosity (HL) LHC perspectives for the ATLAS RPC system, RPC 2014, Tsinghua, Beijing 2014, these proceedings.

[9] A. Polini, Monitoring and Control System of the ATLAS RPCs in view of Run-2, RPC 2014, Tsinghua, Beijing 2014, these proceedings.

[10] E. Pastori, ATLAS RPCs gas system maintenance and improvement, RPC 2014, Tsinghua, Beijing 2014, these proceedings.

[11] A. Polini, Performance and Operation of the ATLAS Resistive Plate Chambers Detector during the 2011 LHC run, PoS(RPC2012)006.

[12] ATLAS Collaboration, Observation of a new particle in the search for the Standard Model Higgs boson with the ATLAS detector at the LHC, Phys. Lett. B 716 (2012) 1-29. 